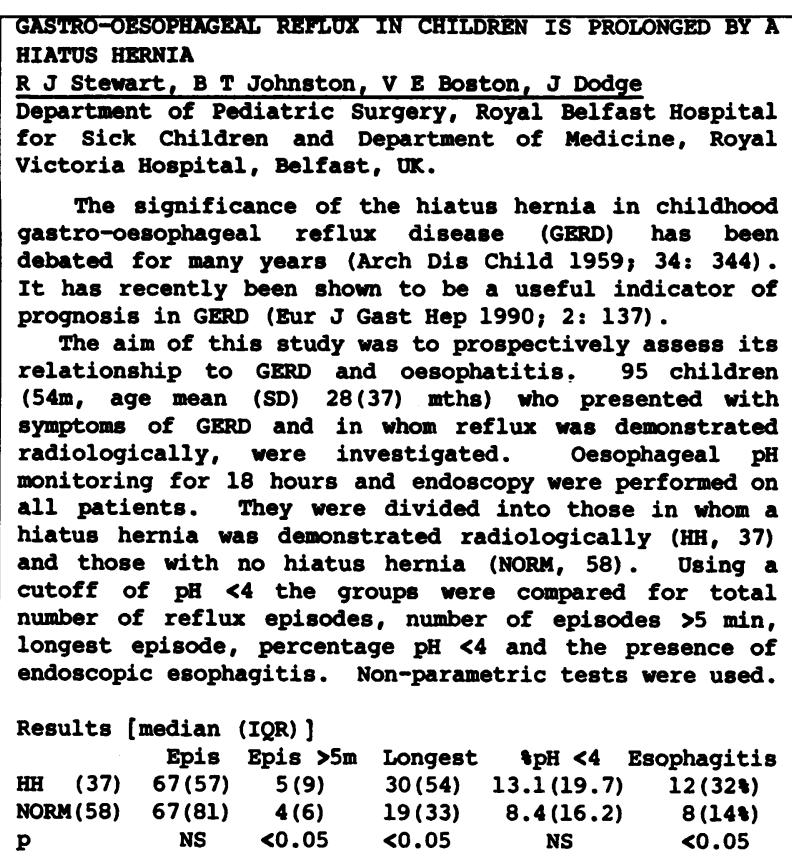

The significance of the hiatus hernia in childhood gastro-oesophageal reflux disease (GRRD) has been debated for many years (Arch Dis Child 1959, 34: 344) It has recently been shown to be a useful indicator of prognosis in GERD (Eur $J$ Gast Hep 1990; 2: 137).

The aim of this study was to prospectively assess its relationship to GERD and oesophatitis, 95 children (54m, age mean (SD) $28(37)$ mths) who presented with symptoms of GERD and in whom reflux was demonstrated radiologically, were investigated. Oesophageal pH monitoring for 18 hours and endoscopy were performed on all patients. They were divided into those in whom a hiatus hernia was demonstrated radiologically (HH, 37) and those with no hiatus hernia (NORY, 58). Using cutoff of $\mathrm{pH}<4$ the groups were compared for total number of reflux episodes, number of episodes $>5$ min, longest episode, percentage $\mathrm{pH}<4$ and the presence of endoscopic esophagitis. Non-parametric tests were used.

Results [median (IQR)]

$\begin{array}{lcclcc} & \text { Epis } & \text { Epis >5m } & \text { Longest } & \text { pH <4 } & \text { Esophagitis } \\ \text { HH (37) } & 67(57) & 5(9) & 30(54) & 13.1(19.7) & 12(328) \\ \text { NORM(58) } & 67(81) & 4(6) & 19(33) & 8.4(16.2) & 8(148) \\ \text { p } & \text { NS } & <0.05 & <0.05 & \text { NS } & <0.05\end{array}$

Both groups had the same number of reflux episodes. However, the number of episodes longer than 5 min was significantly greater in the HH group, as was the duration of the longest episode. The $\mathrm{pH}<4$ data just failed to reach statistical significance $(p=0.06)$. Hiatus hernia was also found to correlate with the presence of esophagitis.

In conclusion, the presence of a hiatus hernia prolongs reflux episodes and is associated with an increased incidence of esophagitis.

\section{Colorectal T150-T158}

T150

\section{FREQUENCY OF HNPCC IN A HEALTH CARE DISTRICT OF NORTHERN ITALY ASSESSED THROUGH A NUCLEAR-PEDIGREE APPROACH. \\ R. Sassatelli, P. Benatti. L, Losi, L, Roncucci, M. Ponz de} Leon. Ist. di Patologia Medica and Ist. di Anatomia Patologica, University of Modena, Modena, ITALY.

The identification of Hereditary Non-polyposis Colorectal Cancer (HNPCC) is difficult, particularly in small pedigrees. Aim of the study was to assess its frequency in the general population through a nuclear pedigree approach, based on the Cancer Registry data. 755 (92.4\%) out of 817 patients recorded in the period 1984-1989 were assessable and stratified according to the presence of six clinical criteria all suggestive of an increased probability of HNPCC: a. vertical transmission of colorectal cancer (CRC); b. aggregation of malignant tumours in the sibship of the proband; c. early age of onset (less than 55 years) of CRC; d. localization of the tumour in the right colon; e. presence of multiple malignant tumours; $f$. mucinous histotype. Five groups were identified: Four or more criteria (41 patients, $5.4 \%)$, Three $(58,7.7 \%)$, Two $(73,9.7 \%)$, One $(203,26.9 \%)$, No criteria $(380,50.3 \%)$. We expanded 25 pedigrees in each group, to second and third degree relatives. HNPCC were then identified according to the definition of the International Collaborative Group on HNPCC. There. were 15 HNPCC out of 25 families in the group with four or more, 6 in the group with three, 3 in the group with two criteria, and none in the group with one criterion $\left(X^{2}=27.6, p<0.01\right)$. Twenty-four families $(3.2 \%)$ were identified as HNPCC (minimal estimate). A nuclearpedigree based approach seems to be a simple and useful tool for the identification of suspected HNPCC. When four or more criteria are identified the expansion of the genealogical tree is mandatory since the probability of HNPCC is consistently high.
PARTIAL OR SUBTOTAL COLECTOMY IN THE TREATMENT OF SLOW TRANSIT CONSTIPATION: A PROSPECTIVE STUDY.

E J R de Graal, E C A M Gillberts, W R Schouten, M van Blankenstein. University Hospital Dykzlgt, Rotterdam, The Netherlands.

Partial colectomy seems to be less beneficial than subtotal colectomy in the surgical treatment of Slow Transit Constipation (STC). However, this finding is based on retrospective studies, in which no segmental this finding is based on retrospective studies, in
colonic transit time (SCTT) studies were performed.

Therefore we conducted a prospective study to evaluate the functional results after partial or subtotal colectomy in patients with STC, based on segmental colonic transit time (SCTT) studies.

From 1985 to 1991227 patients with constipation and defecation disorders were analysed according to a standard protocol. STC was identified in 65 patients. Based on SCTT studies a partial or subtotal colectomy was performed in 35 patients with median follow up of 36 months (range 13 to 68 ). The functional results were scored subjectively (Subi) and objectively (Obi). Recurrent constipation (RC), severe abominal discomfort (SAD) and disabling diarrhoea and fecal incontinence (DDFI) were considered a failure. To evaluate the possible role of anismus in STC electromyography of the pelvic floor and defecography were performed.

The results are listed in the following table:

\begin{tabular}{lcccccc}
\hline \multirow{2}{*}{ Colectomy } & \multicolumn{3}{c}{ Success(\%) } & \multicolumn{3}{c}{ Failure(\%) } \\
& Subj & Obj & & RC & SAD & DDFI \\
\hline Partial $\quad(n=16)$ & 75 & 75 & 13 & 25 & 13 \\
Subtotal $(n=19)$ & 76 & 47 & & 16 & 47 & 16 \\
\hline
\end{tabular}

No correlation was found between anismus and clinical outcome.

CONCLUSIONS:

1. Patients with STC can be treated with partial or subtotal colectomy.

2. The decision to perform a partial or subtotal colectomy in patients with STC can be based on SCTT studies.

3. Anismus has no influence on the final outcome after surgical treatment for STC.
HOW NECESSARY IS COLECTOMY?

C $S$ Probert, $v$ Jayanthi, J F Mayberry.

Leicester General Bospital, Leicester.

The frequency of colectomy for ulcerative colitis in Europeans and South Asians was assessed in a community based 18 year retrospective epidemiological study in Leicestershire. 691 people developed ulcerative colitis during the study period of whom 118 were South Asian. of these patients 85 Europeans and 10 South Asians underwent panproctocolectomy and 4 Europeans and one South Asian ileoanal anastomosis. The annual colectomy rate fell marginally during the study from $5 / 100$ cases in 1974 in Europeans and 10/100 cases in South Asians in the same year. The cumulative colectomy rate did not suffer significantly between the 1970 s and 1980 s in Europeans, bu fell significantly in South Asians $\left(x^{2}=6.3, p<0.05\right)$. The cumulative colectomy rates at 5,10 and 15 iears were 138 (CI 10-16), 18: (CI 13-23) and 218 (CI 12-31) respectively in Europeans and 98 (CI 3-15), 108 (CI 1-20) and 108 (CI 0-23) in South Asians. During the 1980s the duration of disease until colcetomy was significantly longer in South Asians than Europeans $\left(x^{2}=5.3, p<0.05\right)$. Aithough the resection rate in Leicestershire is less than in some other series the overall mortality from ulcerative colitis in the county is not increased and the study clearly suggests that the role of surgery needs to be carefully reassessed and that a high surgical resection rate is unnecessary in ulcerative colitis. 


\begin{tabular}{|c|c|c|c|}
\hline \multicolumn{4}{|c|}{$\begin{array}{l}\text { Although there is some evidence of morphological adaptation of ileal } \\
\text { mucosa following the formation of an ileo-anal reservoir (pouch) it is } \\
\text { unknown whether this is mirrored metabolically. We have therefore } \\
\text { measured the rate of oxidation of metabolic substrates by the mucosa of } \\
\text { established pouches ( } 13 \text { - } 48 \text { months post ileostomy closure) in endoscopic } \\
\text { biopsy samples from } 8 \text { patients with a history of ulcerative colitis (UC). } \\
\text { This was compared with that of normal colon mucosa from } 10 \text { patients with } \\
\text { no history of inflammatory bowel disease and with distal ileal mucosal } \\
\text { biopsies taken from } 4 \text { patients undergoing right hemicolectomy for cancer } \\
\text { of the ascending colon. } \\
\text { Each biopsy specimen ( } 4 \text { - } 15 \text { mg wet weight) was assayed for the } \\
\text { conversion of radiolabelled glucose, glutamine or butyrate to }{ }^{14} \mathrm{CO}_{2} \text { in a } \\
\text { medium containing all three substrates. The values of n refer to biopsy } \\
\text { specimens analyzed. Some patients provided multiple specimens, whilst in } \\
\text { other cases results were not obtained for each substrate. Significance of } \\
\text { differences was assessed at the } 95 \% \text { confidence interval. }\end{array}$} \\
\hline \multicolumn{4}{|c|}{ Substrate oxidation - pmoles/mg wet weight/hour Mean \pm sem ( $n$ ) } \\
\hline & $\begin{array}{l}\text { Glucose } \\
(10 \mathrm{mM})\end{array}$ & $\begin{array}{l}\text { Glutamine } \\
(10 \mathrm{mM})\end{array}$ & $\begin{array}{l}\text { Butyrate } \\
(10 \mathrm{mM})\end{array}$ \\
\hline Normal Colon & $305 \pm 21(29)$ & $1650 \pm 239(35)$ & $26070 \pm 2800(35)$ \\
\hline Normal Ileum & $428 \pm 123(5)$ & $7790 \pm 1216(7)$ & $27800 \pm 8570(6)$ \\
\hline UC Pouch & $361 \pm 100(13)$ & $2249 \pm 476(12)$ & $17380 \pm 2320(10)$ \\
\hline \multicolumn{4}{|c|}{$\begin{array}{l}\text { There were no significant differences in the utilisation of glucose and } \\
\text { butyrate between the three groups. The flux of glutamine to } \mathrm{CO}_{2} \text { was found } \\
\text { to be significantly higher in ileum than in colon, in keeping with published } \\
\text { data. Glutamine utilisation by pouch mucosa was significantly less than in } \\
\text { ileum and not significantly different from colon. These results provide the } \\
\text { first demonstration that a metabolic accommodation of the pouch mucosa } \\
\text { to a colon-like milieu may accompany the morphological changes which } \\
\text { have previously been observed. }\end{array}$} \\
\hline
\end{tabular}

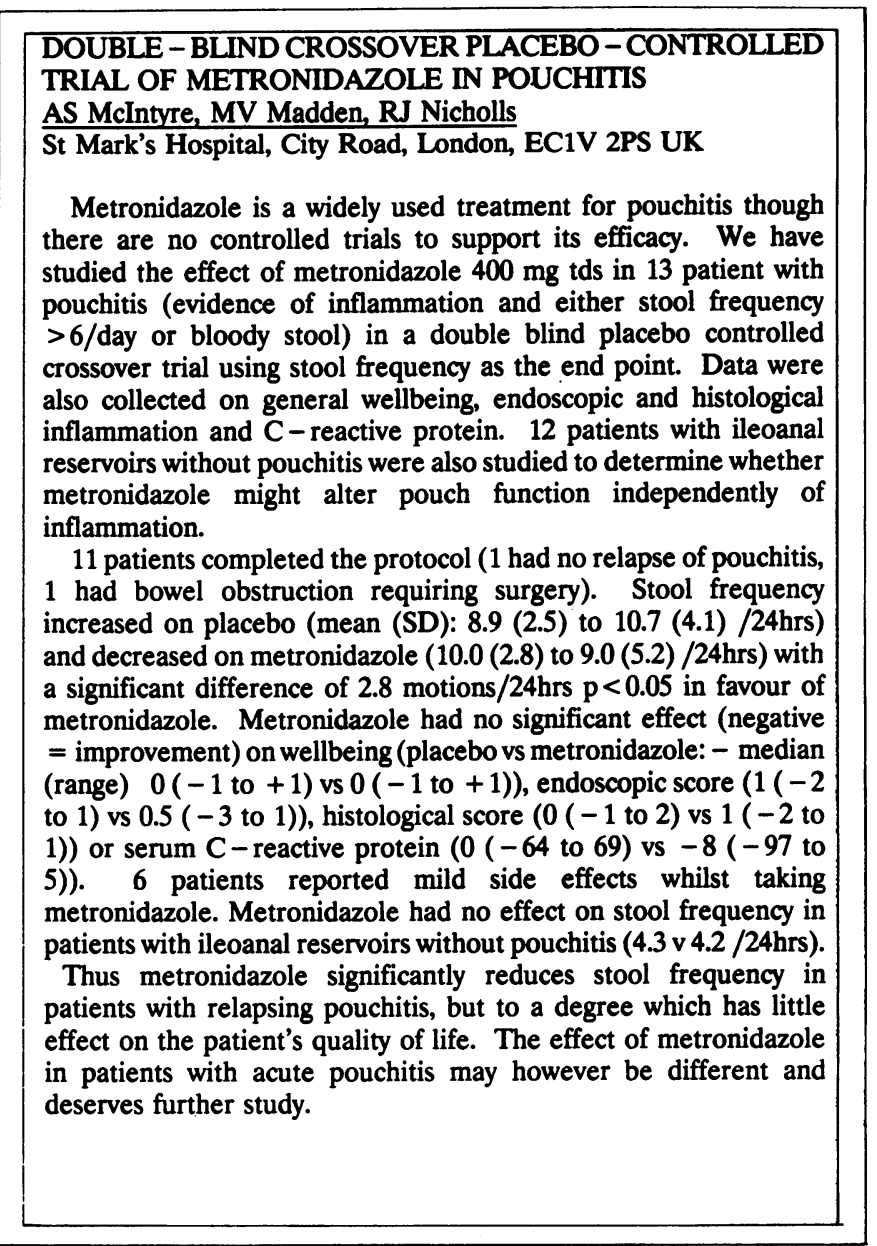

THE DISTRIBUTION OF INFLAPYATORY, MORPHOLOGICAL AND METAPLASTIC CHANGES IN THE PELVIC ILEAL RESERVOIR M A Shepherd, C J Healey, B F Warren, P I Richman, W H T Thomson, S P Wilkinson

Departments of Histopathology, Gastroenterology and Surgery, Gloucestershire Royal Hospital, Gloucester and Departments of Histopathology, Bristol Royal Infirmary and Mount Vernon Hospital, Northwood.

The mucosa of the pelvic ileal reservoir undergoes adaptive changes on exposure to the faecal stream: these include inflammabory, architectural and metaplastic changes. There have been no forwal studies of the distribution of such changes. We have studied 23 quadruple loop ileal pouches, constructed for ulcerative colitis (20 pts) and FAP ( 3 pts). No patient fulfilled criteria for pouchitis. Standard duplicated biopsies were taken from the proximal limb (5 to $10 \mathrm{cms}$ ), the anterior wall, the posterior wall and the body of the reservoir. Using an established scoring system, there was a highly significant increase in inflammatory scores in posterior wall biopsies compared with the anterior wall. These results suggest that the adaptive changes are the direct result of contact with static faecal contents. Only one patient showed significant inflammation in the proximal limb. There was no evidence of mucosal prolapse in the anterior wall biopsies. Colitic patients showed substantially more inflammatory and architectural changes than those with FAP. Over half of the pouches showed colonic characteristics as defined by mucin histochemical and PR $3 A 5$ immunohistochemical studies. The distribution of these colonic phenotypic changes was not significantly different at any site within the reservoir. Our studies add further weight to the concept of colonic metaplasia in the pouch but demonstrate that these changes are focal and are likely to be the result of direct contact with static faecal residue. They also demonstrate that a single randow biopsy of pouch mucosa is of limited value in assessment of pathological changes and for screening for potential neoplastic change within the reservoir.
CUTTING SETON - AN ANCIENT BUT EFFECTIVE TREATMENT FOR COMPLEX HIGH FISTULA-IN-ANO. J.S. McCourtney, I.G. Finlay

Royal Infirmary, Castle Street, GLASGOW, G4 05F

Eradicating a complex high fistula-in-ano with preservation of faecal continence remains a difficult surgical challenge. The traditional approach of using a cutting seton with division of the internal sphincter results in a high risk of faecal incontinence. We report 15 patients ( 7 males) with a high fistula-in-ano treated by a cutting seton technique without preliminary internal sphincterotomy.

Thirteen patients had undergone surgery elsewhere (median number of operations 3 , range $0-19$ ), with a median duration of symptoms of 2 years (range $0.1-21$ years). The fistulae were classified as high: transsphincteric (4), transsphincteric with complex extension (7), extrasphincteric (1) and rectovaginal (3). After drainage of sepsis a seton (silk ligature) was tied around both the internal and external anal sphincter below the fistula and tightened at monthly intervals.

The fistula was cured in 13 patients $(87 \%)$ requiring a median number of operations of 3 (range 2 - 6). After treatment 1 patient $(6 \%)$ was incontinent of faeces; a further $3(20 \%)$ had minor leakage.

The data suggest that cutting seton is an effective treatment for complex high fistula-in-ano. The avoidance of preliminary internal sphincterotomy may explain the relatively low incidence of minor incontinence. 
POUCH-VAGINAL FISTULA

JS Groom, RJ Nicholls, PR Hawley, RKS Phillips

St Mark's Hospital, City Road, London ECIV 2PS

Between 1976 and June 1991, 161 females underwent restorative proctocolectomy. Sixteen (9.9\%) developed pouchvaginal fistula. No difference between inflammatory $14 / 139$ (10.1\%) and non-inflammatory $2 / 22$ (9.1\%) disease, severity of colitis, or number of stages in operation. All fistula involved the ileo-anal anastomosis. There was no significant difference between hand sewn $9 / 117(7.6 \%)$ and stapled $7 / 44$ (15.9\%) ileo-anal anastomosis, although 3/15 (20\%) had doublestapled anastomosis. Of the 16,8 had pelvic sepsis in the immediate post operative period, only 3 had an anastomotic defect and one who catheterizes had a stricture. In 6 cases there was no apparent reason for PVF. There was no case of Crohn's disease. Three developed PVF before ileostomy closure, 13 after a median interval of 7.5 months (range 0.5 to 33 months).

Five further patients were referred giving a total of 21 cases These were divided into group 1 with PVF developing before ileostomy closure ( 5 patients) and group 2 with development after (16 patients). The procedures and outcome in group 1 spontaneous closure $(n=2)$, direct repair $(n=2$, closure 1$)$ pouch excised $(n=1)$ and unhealed $(n=1)$. For group 2: spontaneous closure $(n=2)$, ileostomy alone $(n=7$, closure 1$)$, Ileostomy with; direct repair $(n=4$, closure 1$)$, pouch advancement flap $(n=2$, closure 1$)$, revision ileo-anal anastomosis ( $n=2$, closure 0 ), fistulotomy with delayed direct repair ( $n=1$, outcome awaited). In this group excision of the pouch was necessary in 6 cases. With these small numbers the prognosis appears to be worse when PVF occurs after ileostomy closure. Optimal management is not yet established.

Radiation carcinogenesis is greatly enhanced at colonic anastomoses. With chemical carcinogens, sutures enhance anastomotic tumour formation preceded by elevated cellular proliferation. This study was after irradiation.

Method

60 adults male F344 rats were used. In 30 animals the lower descending colon and rectum was exposed to 16 $G y$ orthovoltage $x$-rays, via anterior and posterior portals, through a $2 \times 1 \mathrm{~cm}$ aperture in a $4 \mathrm{~mm}$ lead sheet. One week later all 60 animals underwent a $5 \mathrm{~mm}$ transverse colotomy within the radiation field, which was repaired with 1)silk, stainless steel or vicryl sutures. 30 animals were killed after 4 weeks and a further 30 after 3 months. technique.

\section{Results}

CCPR was significantly elevated at sutured anastomoses compared to adjacent descending colon in both (See Table 1). Elevated proliferation was more (hee irradiated compared to the nonpronounced at the irratiat anastomosis at 4 weeks (p<0.05, $f=4.3)$ and 13 weeks $(p<0.005, f=14.1)$. Little difference was noted between the suture types.

\section{Conclusion}

Anastomotic proliferative changes are exaggerated by irradiation. This has potentially disturbing implications in view of the increasing use of adjuvant radiotherapy for rectal carcinoma.
CHILDHOOD LIVING CONDITIONS DETERMINE HELICOBACTER PYLORI SEROPOSITIVITY IN ADULT LIFE, MA Mendall. PM Goggin. Nicola Molineaux. Joanne Levy,

\section{EToosy@.D Strachan*.TC Northfield.}

Departments of Medicine and Public Health Sciences*, St. Georges

Hospital Medical School and The Practice, Wallington@, London.

Background. H. pylori has emerged as a major cofactor in peptic ulcer disease and gastric cancer. Studies into risk factors for infection have concentrated on current living circumstances and have shown an association with social class. Our aim was to see whether childhood living circumstances are a risk factor for infection with $\mathrm{H}$. pylori.

Methods An in-house Elisa using a partially purified antigen was validated on 227 subjects (median age 46, 55\% positive for $H$. pylori on biopsy), and gave a sensitivity of $127 / 130$ (98\%) and specificity of $87 / 93$ (94\%) using a cut-off point midway between two clearly defined populations of positives and negatives. A bimodal distribution was also obtained in the main study and the trough corresponded to that in the validation study. 215 subjects (age 18-82, median 46), attending a health screening clinic in London were interviewed and serum taken for serology. The results were analysed by multiple logistic regression using GLIM.

Results.

Seropositivity varied from $9 \%$ (age $<30$ ) to $67 \%$ (age $70+$ ). In childhood, absence of a fixed hot water supply ( $\mathrm{p}=0.0005)$ and domestic crowding $(p=0.0005)$ were powerful independent risk factors for current infection with $\mathrm{H}$. pylori. Amongst current living conditions, only the number of children living in the household was independently associated with $\mathrm{H}$. pylori infection $(\mathrm{p}=0.004)$.

Conclusions

Most British adults currently infected by $\mathrm{H}$. pylori probably acquired their infection by household contact in childhood. Improved childhood living circumstances with a decrease in prevalence of $\mathrm{H}$. pylori is probably the mechanism behind the cohort effects observed in mortality from peptic ulcer disease.

PREVALENCE OF HELICOBACTER PYLORI (HP) INFECTION IN PATIENTS WITH PRECANIEROUS CHANIGES AND GASTRIC CANCER. F.Farinati, F.Valiante, B.Germana, R. Baffa, M.Rugge, G.Della Libera, MC.Fanton, F. Vianel10, F.Di Mario, R. Naccarato. Catt.Mal att. App.Dig., Anat. Pat., II I0 Catt., Padova, Italy. Several recent papers have suggested a role for HP infection with respect to gastric cancer development. The aim of this study was to evaluate the prevalence of $H P$ infection in an endoscopic population of patients with gastric precancerous conditions and lesions. Perendoscopic biopsies (antral and fundic) from $186 \mathrm{pts}$ (112 M, $74 \mathrm{~F}$, mean age 52 yrs, range 21-84) were evaluated, recording the presence and degree of HP infection: chronic superfilial gastritis (CSG) chronic deep (or pre-atrophic) gastritis (CDI), Ehronic atrophic gastritis (CAli), intestinal metaplasia (IM), epithelial dysplasia (ED), cancer (K) and, as a further control, duodenal ulcer pts (DU) were investigated. In the population as a whole, HP infertion rate increased with age. For CSG a higher prevalence was found in the fundic area and for $\mathrm{CDF}$ in antral $(80 \%$ and $76 \%$ respectively) ( $p<0.005)$. A significantly lower prevalenice was observed in CAIS $(50 \%, p<0.002)$, particularly in the antral type (39\%, $\mathrm{p}<0.001)$. Also the severity of colonization was significantly lower in CAS pts $(p=0.01)$. HF infection rate was also lower in both IM and $E D$ patients $(p<0.000)$. None of the K pts had HF in the eancerous tissue, and only $23 \%$ were HP+ in adjacent mucosa. DU pts had an $80 \%$ infection rate in both antral and fundir mucosa. In our experience, HP infection inversely correlate with the presence of gastric precancerous changes and cancer $(p=0.000)$. A cautious interpretation of the epidemiological data regarding HP infection and gastric cancer therefore is, in our opinion, mandatory, also because DU pts (who are most frequentiy infected) are notoriously at a very low $r$ isk for sancer. 